\section{Globalization of sponsored clinical trials: reply}

\author{
Fabio A. Thiers, Anthony J. Sinskey and Ernst R. Berndt
}

Dr Karlberg's correspondence outlines an alternative way of analysing data provided by ClinicalTrials.gov. His results are generally similar to ours, and any differences can be explained by the use of distinct inclusion criteria and formulae for the calculation of trial participation growth rates of individual countries.

Trials included in our analysis were industry-sponsored biopharmaceutical clinical trials that were either completed or actively recruiting on 12 April 2007. These trials were selected when a biopharmaceutical company was listed in the "Information Provided By:" field in the ClinicalTrials.gov web site. The field only allows the listing of one entity, which corresponds to the organization that is responsible for the accuracy of the information and serves as the main sponsor of the trial. As the focus of the study was on the global dispersion of trial of drugs and biologics, we did not include trials of medical devices, diagnostics, or observational studies. We also did not include "no longer recruiting" or "terminated" industry trial descriptions because in our previous extensive non-automated analysis of ClinicalTrials.gov these entries were found to be less accurate in the reporting of site location information. It appears to us that Dr Karlberg incorporated all trials labelled as industrysponsored by ClinicalTrials.gov, including trials in which biopharmaceutical companies were collaborators with governments and universities, and trials involving medical devices and diagnostics, observational studies, and no longer recruiting studies. Thus, the sample of trials from Dr Karlberg is larger than ours.

The calculation of the weighted average relative annual growth rate (ARAGRs) in shares between 2002-2006 was based on allocation by date of start of recruitment, not date at which the trial was registered at the ClinicalTrials.gov web site. We reasoned that date of start of recruitment better reflected the temporal evolution of the actual decisions made by planners of global trials, which are the behavioural events we tried to capture with our analysis. For our purposes, date of registration is less meaningful, as trials with later registration dates frequently had initiated recruitment in previous years. We used data from both actively recruiting and completed trials in the calculation of the ARAGRs, and in the calculation of average trial capacity (number of sites per trial) of individual countries. The calculation of trial density and current shares was based only on actively recruiting sites as of 12 April 2007, which numbered 3,123 trials in 74,480 sites (sum of top $50=73,882$ ). The number of actively recruiting sites for each of the top performing countries were presented in Figure 1 and in Tables 1, S3 and S4. By comparison, Dr Karlberg quantified temporal changes in trial participation for all types of trials registered between October 2005/
September 2006 and October 2006/September 2007. We constructed a time series (2002; $2003 ; 2004 ; 2005$; 2006) based on date of start of recruitment, and computed the average of annual growth rates by weighting the number of sites initiating recruitment in the second year of each growth rate $(8,688$ in 2003, 15,662 in 2004, 24,730 in 2005 and 41,118 in 2006), thereby compensating for increased registry coverage over time. Since our data sets and the periods used in our analyses differ somewhat from those of Dr Karlberg, we are not surprised that our respective results involving the global distribution of clinical trials are slightly different, albeit very similar qualitatively. We did not calculate changes of position in the ranking of growth rates, and thus those findings are not comparable to ours.

The same basic conclusions of our paper can be derived from Dr Karlberg's correspondence, namely: first, clinical trials are still primarily performed in traditional nations; second, many (but not all) of these traditional nations experienced negative growth in their relative participation (experienced share declines) between 2002-2006; third, individually, emerging economies are still relatively small players; fourth, in terms of number of sites, emerging economies are growing more rapidly than are traditional countries; and fifth, a substantial and increasing proportion of trials are being conducted in emerging regions. Where there are differences, they should be expected given the different conventions used regarding timing and definition of industry-sponsored biopharmaceutical trials.

Fabio A. Thiers, Anthony J. Sinskey and Ernst R. Berndt are at the MIT Center for Biomedical Innovation, 77 Massachussetts Avenue, Cambridge, Massachusetts 02139, USA.

e-mail: eberndt@mit.edu 